\title{
The pattern of the arterial supply of the pancreas in Anthropoid apes, catarrhine monkeys and platyrrhine monkeys
}

\author{
By
}

\author{
Alimujiang SHAWUTI, Takayoshi MIYAKI, Toshiyuki SAITO, Masahiro ITOH \\ Department of Anatomy, Tokyo Medical University, 6-1-1 Shinjuku, Shinjuku-ku, Tokyo 1608402 Japan \\ -Received for Publication, September 4, 2009-
}

\begin{abstract}
Key Words: pancreas, celiac artery, superior mesenteric artery, anthropoid, monkeys
Summary: To get the full understanding of the arterial distribution to the pancreas, the analysis of the distribution of the variety of monkey species would be helpful. In this study, we studied the layout of the pancreatic artery in anthropoids (1 gorilla, 3 chimpanzees and 2 white-handed gibbons), in catarrhine monkeys (1 hamadryas baboon, 2 anubid baboons, 10 savannah monkeys) and in platyrrhine monkeys (6 squirrel monkeys).

The pancreas of the monkeys was supplied by the arteries originating from the celiac trunk and/or superior mesenteric artery. There were three patterns in the arterial distribution; (1) the celiac artery supplied the major area of the pancreas. (2) the superior mesenteric artery supplied the major area of the pancreas. (3) the celiac artery supplied the whole pancreas. The pattern of the arterial distribution to the monkey pancreas had a wide variety. The result would be helpful for the elucidation of the development of the vascular distribution in the pancreas.
\end{abstract}

\section{Introduction}

The arterial distribution to the pancreas has been vigorously studied in human adults (Martin II KG et al., 2009; Gaujoux S et al., 2009; Kimura S et al., 2007). In the human fetuses, Sakagami S and Akaiwa J recently studied the distribution to the pancreas in the human fetus (Sakagami S, 1989, Akaiwa J, 1984). However, the reports for other animal species are rarely found. To our knowledge, there is no precise description of the arterial distribution in the pancreas in primates (Swindler DR et al., 1973). We think that the study of the distribution in the primates may provide precious information about the development of the artery. Here, we compared the arterial distribution in different primates. We used 6 anthropoid apes, 13 catarrhine monkeys, and 6 platyrrhine monkeys. We report here the difference in the arterial distribution according to the species.

\section{Material and Methods}

We used one gorilla (species: Gorilla), three chimpanzees (species: Pan troglodytes) and two white-handed gibbons (species: Hylobates lar) in the anthropoid apes (Anthropoidae). We also used one hamadryas baboon (species: Papio hamadryas) and two anubis baboons (species: Papio anubis) and ten savannah monkeys (species: Cercopithecus aethiops) in the catarrhine monkeys (Catarrthinae: the old world monkeys). We also used six squirrel monkeys (species: Saimiri) in the platyrrhine monkeys (Platyrrhinae: the new world monkeys). In regard to the classification of the primates, we referred to the report by Iwamoto and Uchida (Table 1) (Iwamoto, 1985, 1987; Uchida, 1998).

After we removed all abdominal organs from the body as one specimen, we examined the layout of the pancreas and the arterial supply to the pancreas. Samples were stored in $50 \%$ alcohol after they were stabilized with $10 \%$ formalin. The gorilla was given by Dr. H Kumakura of Osaka University Graduate School of Human Sciences. Almost all the primates subjected in this study were given from the Kyoto University Primates Research Institute between 1992 and 1994.

\section{Nomenclature}

In the apes, neither the name of the part of the pancreas, nor the name of the vessels around the pancreas are well registered. We needed to adopt some nomenclature of the pancreas for our study on the pancreas.

In other animals, there are the names of the right and left lobes, pancreatic body and pancreatic annule in the 


\begin{tabular}{|c|c|c|c|c|}
\hline \multicolumn{4}{|c|}{ Classification of the Apes } & $\begin{array}{c}\begin{array}{c}\text { Subjects } \\
\text { No }\end{array} \\
\text { (n) }\end{array}$ \\
\hline \multirow{3}{*}{\begin{tabular}{l}
$\frac{0}{\pi}$ \\
$\frac{0}{0}$ \\
\hdashline \\
0 \\
0 \\
0 \\
$\frac{0}{2}$ \\
$\frac{1}{2}$ \\
$\frac{1}{<}$
\end{tabular}} & \multirow{2}{*}{ 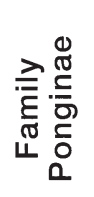 } & \multicolumn{2}{|r|}{$\begin{array}{l}\text { Gorilla } \\
\text { (Gorilla) }\end{array}$} & 1 \\
\hline & & \multicolumn{2}{|c|}{$\begin{array}{c}\text { Chimpanzee } \\
\text { (Pan troglodytes) }\end{array}$} & 3 \\
\hline & ᄂ: & \multicolumn{2}{|c|}{$\begin{array}{l}\text { White - handed gibbon } \\
\text { (Hylobates lar) }\end{array}$} & 2 \\
\hline \multirow{3}{*}{ 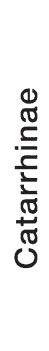 } & \multirow{3}{*}{ 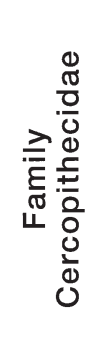 } & \multirow{2}{*}{$\frac{0}{\frac{0}{\pi}}$} & $\begin{array}{l}\text { Hamadryas baboon } \\
\text { (Papio } \\
\text { hamadryas) }\end{array}$ & 1 \\
\hline & & & $\begin{array}{l}\text { Anubis baboon } \\
\text { (Papio } \\
\text { anubis) }\end{array}$ & 2 \\
\hline & & 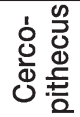 & $\begin{array}{l}\text { Savannah } \\
\text { monkey } \\
\text { (C. aethiops) }\end{array}$ & 10 \\
\hline 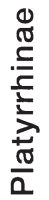 & 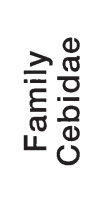 & & $\begin{array}{l}\text { Squirrel } \\
\text { monkey } \\
\text { (Saimiri) }\end{array}$ & 6 \\
\hline
\end{tabular}

Table 1. F.: Family. The upward arrow on the left side means the direction of the development.

Nomina Anatomica Veterinaria Japonica and in the Comparative Anatomy of the Domestic Animals (in Japanese) (J Society of Veterinary Anatomists, 1978; Kato Y, 1964). We did not see any right or left lobes in the monkeys. Instead, we found that all apes had the pancreas structure just like that of a human. Therefore, we decided to adopt the names of the nomina anatomica for humans (Fig. 1) (Miyaki, 2000; Standring S et al., 2008). In regard to the pancreas body and the tail, we adopted the conventional delineation to separate them evenly as it is still used in surgery (Kimura S, 2007). There is no obvious border between the pancreas body and its tail.

\section{Results}

I The arterial supply to the pancreas in Anthropoidae.

1. The arterial supply to the pancreas in gorillas.

With our study on one gorilla, we found that the arterial supply to the pancreas in the gorillas was from the celiac artery and from the superior mesenteric artery (Fig. 2). The celiac artery delivered the common hepatic, the left gastric and the splenic arteries. The common hepatic artery delivered the right gastroepiploic artery, while the splenic artery delivered the left gastroepiploic artery.

The head part of the pancreas of this gorilla was sup-

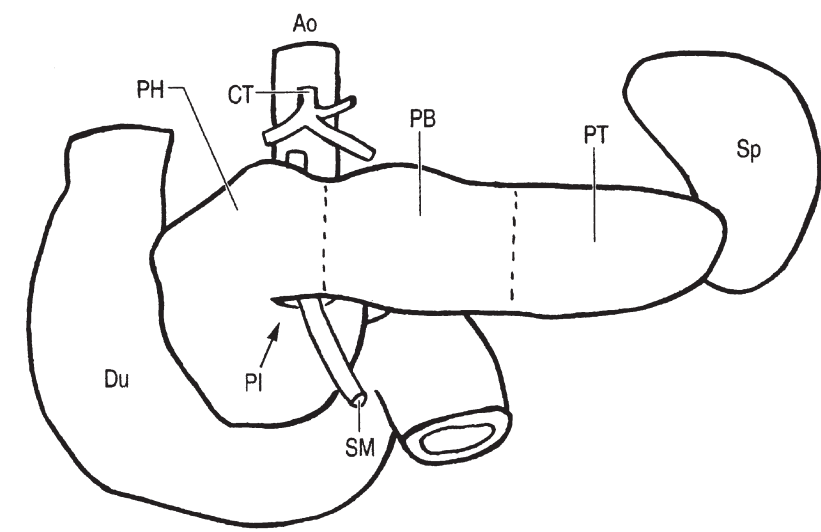

Fig. 1. The nomenclature of the human pancreas (From Miyaki, 2003). PH: pancreas head, PB: pancreas body, PT: pancreas tail, PI: pancreatic notch, Sp: spleen, SM: superior mesenteric artery.

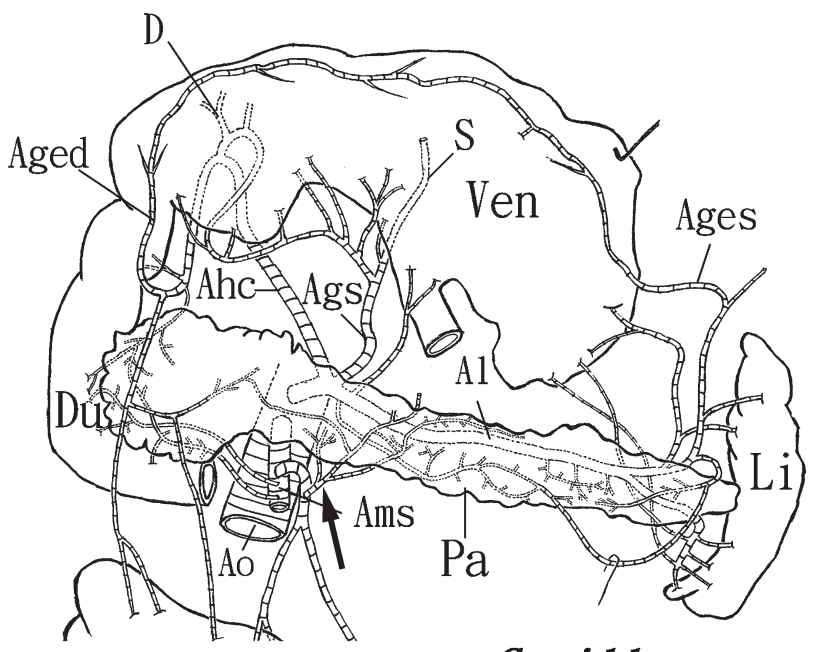

Gorilla

Fig. 2. The arterial distribution to the pancreas of the gorilla. After the gorilla's stomach was inverted up, the posterior surface of the stomach and the anterior surface of the pancreas are seen. The pancreas was supplied from the celiac trunk and the superior mesenteric artery.

Agd: Arteria gastrica dexter, Aged: Arteria gastroepiploica dexter, Ages: Arteria gastroepiploica sinister, Ags: Arteria gastrica sinister, Ah: Arteria hepatica, Ahc: Arteria hepatica communis, Al: Arteria lienalis, Ams: Arteria mesenterica superior, Ao: Aorta, D: Arteria hepatica dexter, Du: Duodenum, Li: Lien, M: Arteria hepatica media, Pa: Pancreas, S: Arteria hepatica sininster, Tc: Truncus coeliacus, Ven: Ventriculus.

The words shown in this figure are common abbreviation throughout this article.

plied by one artery originating from the common hepatic artery and two arteries originating from the superior mesenteric artery. The pancreas body and tail were supplied by the branches from the splenic artery, while a part of 

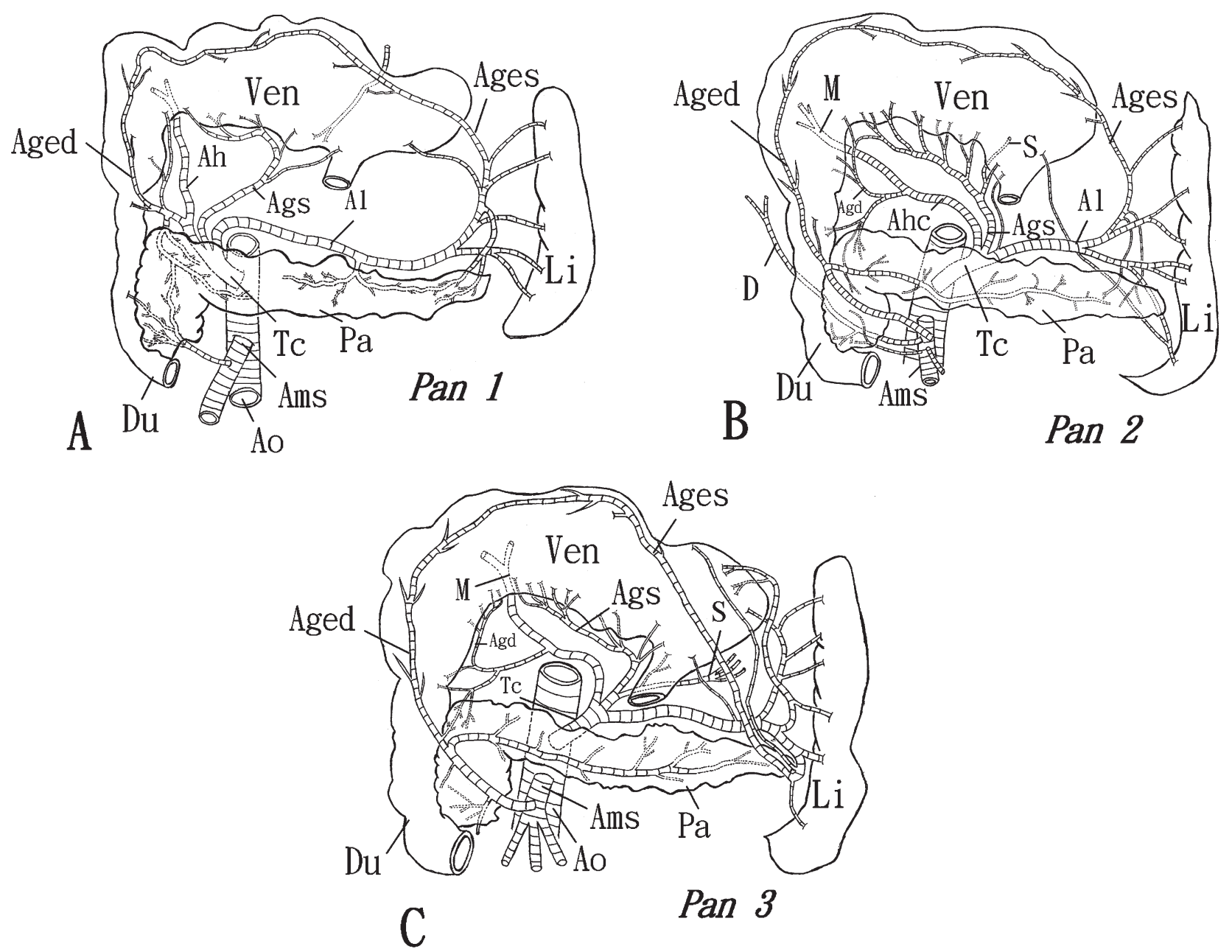

Fig. 3. The arterial distribution to the pancreas of the chimpanzees.

A: The pancreas received blood supply from the celiac and the superior mesenteric arteries. The region supplied by the superior mesenteric artery was minor.

B: The right gastroepiploic artery originated from the superior mesenteric artery. While the pancreas received blood supply from the celiac and the superior mesenteric arteries, the region by the celiac artery was minor. A part of the pancreas head and tail received the supply from the celiac artery.

C: In this case, the right gastroepiploic artery originated from the superior mesenteric artery. A part of the pancreas head received blood supply from the celiac artery. The region supplied by the celiac artery was very minor.

the pancreas body was supplied by a branch of the superior mesenteric artery (arrow).

2. The arterial supply to the pancreas in chimpanzees.

In chimpanzees, we found that the arterial supply to the pancreas originated from the celiac and the superior mesenteric arteries.

The first case (Fig. 3-A): the celiac artery in this chimpanzee divided into three arteries; the common hepatic, the left gastric artery and the splenic artery. The right gastroepiploic artery originated from the common hepatic artery. The left gastroepiploic originated from the splenic artery.
The head of the pancreas had the blood supply from the common hepatic artery and from the superior mesenteric artery. The major part of the pancreas body had the blood supply from the common hepatic artery. The rest of the pancreas body and the pancreatic tail received the blood supply from the splenic artery.

The second case (Fig. 3-B): In this case too, the celiac artery branched into the three arteries; the common hepatic artery, the left gastric artery and the splenic artery. In this case, the right gastroepiploic artery originated from the superior mesenteric artery and the left gastroepiploic artery originated from the splenic artery.

The head of the pancreas had the blood supply from 
the common hepatic artery, and from the superior mesenteric artery. In regard to the pancreatic tail, a part of it was supplied by the splenic artery. The remaining major area of the pancreas was supplied by the superior mesenteric artery through the right gastroepiploic artery (Fig. 3-B).

The third case: The celiac artery was found to have delivered the common hepatic, the left gastric and the splenic arteries. The right gastroepiploic artery originated from the superior mesenteric artery while the left gastroepiploic originated from the splenic artery.

A small part of the pancreas head had the blood supply from the common hepatic artery. The remaining major part of the pancreas was supplied by the right gastroepiploic artery from the superior mesenteric artery (Fig. 3-C).

3. The arterial supply to the pancreas in the white-handed gibbons (Hylobates).

In the hylobates, the right gastroepiploic artery originated from the common hepatic artery, and the left gastroepiploic artery originated from the splenic artery.

The first case: the celiac artery divided into the common hepatic, left gastric and the splenic artery. The pancreas head had a blood supply through the branches from the right gastroepiploic artery and the superior mesenteric artery. The pancreas body and the tail had the blood supply from the splenic artery (Fig. 4).

The second case: in this case, the celiac artery had the direct branch to the pancreas head as well as the triplication to the splenic artery, left gastric and the common hepatic artery. The pancreas head had the blood supply from the direct branch and from the common hepatic artery. The pancreas body and the tail had the blood supply from the splenic artery (Fig. 4).

\section{The arterial supply to the pancreas in the catarrhine monkeys.}

1. The arterial supply to the pancreas in Papio hamadryas.

The celiac artery divided into the splenic, the left gastric, the common hepatic and the hepatica propria artery in the papio hamadryas. The right gastroepiploic artery originated from the superior mesenteric, and the left gastroepiploic originated from the splenic artery (Fig. 5).

The blood supply to the pancreas was produced by the celiac artery and the superior mesenteric artery. The pancreas head was supplied by the three branches from the common hepatic artery and the superior mesenteric artery. The pancreatic body and the tail was supplied by the branches from the splenic artery.

2. The arterial supply to the pancreas in Papio anubis.

The celiac artery divided into the splenic, the left gastric and the common hepatic artery. The right gastroepiploic artery originated from the superior mesenteric artery. The left gastroepiploic artery originated from the splenic artery (Fig. 6).

The first case: the branches, which originated from the common hepatic artery, supplied to the pancreas head and the body. The pancreas tail was supplied by the branches directly from the celiac artery and the branch from the splenic artery (Fig. 6-A).

The second case: the branches from the superior mesenteric artery and from the common hepatic artery
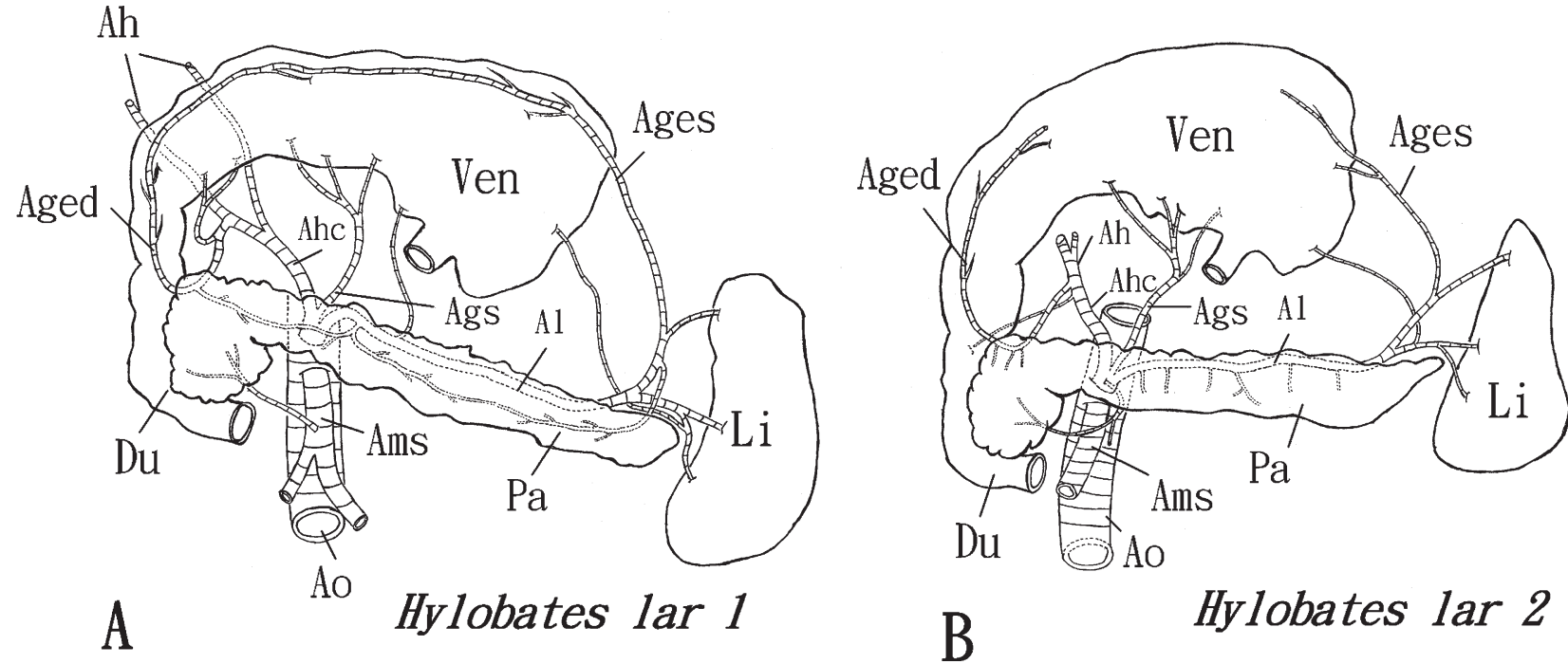

Fig. 4. The arterial distribution to the pancreas in the hylobates lar (white-handed gibbon). 4-A: The pancreas received the blood supply from both the celiac and the superior mesenteric arteries. But the region supplied by the superior mesenteric was small. 4-B: The whole pancreas had the blood supply from the celiac artery. 


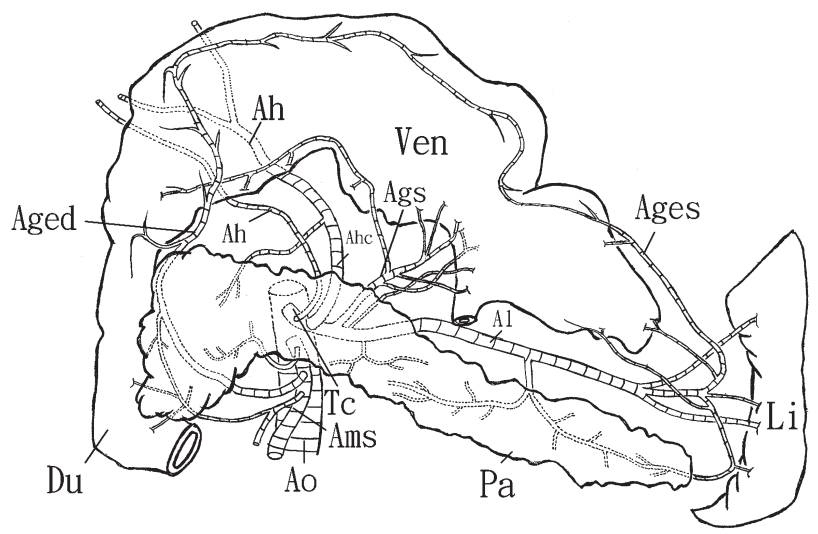

P. hamadryas

Fig. 5. The arterial distribution to the pancreas of the hamadryas monkeys.

The right gastroepiploic artery originated from the superior mesenteric artery. The pancreas received blood supply from both celiac artery and the superior mesenteric artery. But the region of the superior mesenteric artery was not large.

distributed to the pancreas head. The branches from the celiac artery and the splenic artery distributed to the pancreas body and the tail (Fig. 6-B).

3. The arterial supply to the pancreas in Cercopithecus aethiops.

The celiac artery divided into the splenic, the left gastric and the common hepatic arteries. The right gastroepiploic originated from the common hepatic artery.
The left gastroepiploic originated from the splenic artery (Fig. 7). The arterial distribution to the pancreas in the ten subjects could be classified into two groups. (1) The branches originated from the celiac artery and from the superior mesenteric artery supplied the pancreas (in 9 cases). The pancreas head was supplied by the superior mesenteric artery and the common hepatic artery. The pancreas body and the tail were supplied by the splenic artery (Fig. 7-B). (2) Only the celiac artery supplied to the pancreas (one case). The pancreas head and the tail was supplied by the common hepatic artery. A part of the pancreas body/tail part was supplied by the splenic artery. (The splenic artery supplied the pancreas body and tail by the direct communicating vessel or via the left gastroepiploic artery, Fig. 7-A).

\section{The arterial supply to the pancreas in the platyrrhine monkeys.}

(the arterial supply to the pancreas in the squirrel monkey, saimiri.)

After we studied the branching of the celiac artery in six squirrel monkeys, we found that it could be classified into three groups. (1) The celiac artery divided into the left gastric, the splenic and the common hepatic arteries (in two out of six cases). (2) The celiac artery divided into the left gastric, splenic and the common hepatic arteries. But, the celiac artery further had another branch to the right adrenal gland (in three out of six cases). (3) The celiac artery divided into the left gastric and the splenic arteries. The common hepatic artery originated from the superior mesenteric artery (in one case).

Among the six saimiris, the right gastroepiploic artery originated from the common hepatic artery, and the left

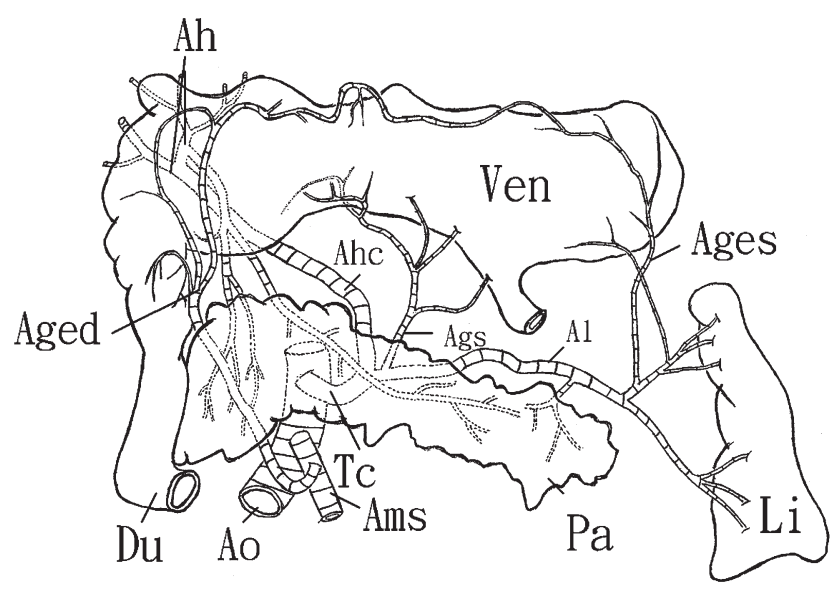

A

P. anubis 1

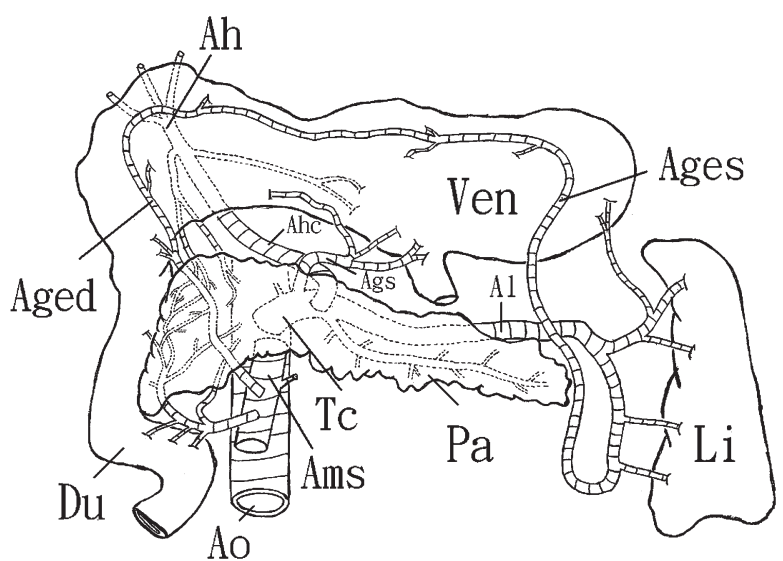

B
P. anubis 2

Fig. 6. The arterial distribution to the pancreas of the pan anubis. 6-A: the right gastroepiploic artery originated from the superior mesenteric artery. In this pan anubis, whole pancreas received blood supply from the celiac artery. 6-B: the right gastroepiploic artery originated from the superior mesenteric artery. In this anubis, the pancreas was supplied by both the celiac and the superior mesenteric arteries although the region supplied by the superior mesenteric was small. 

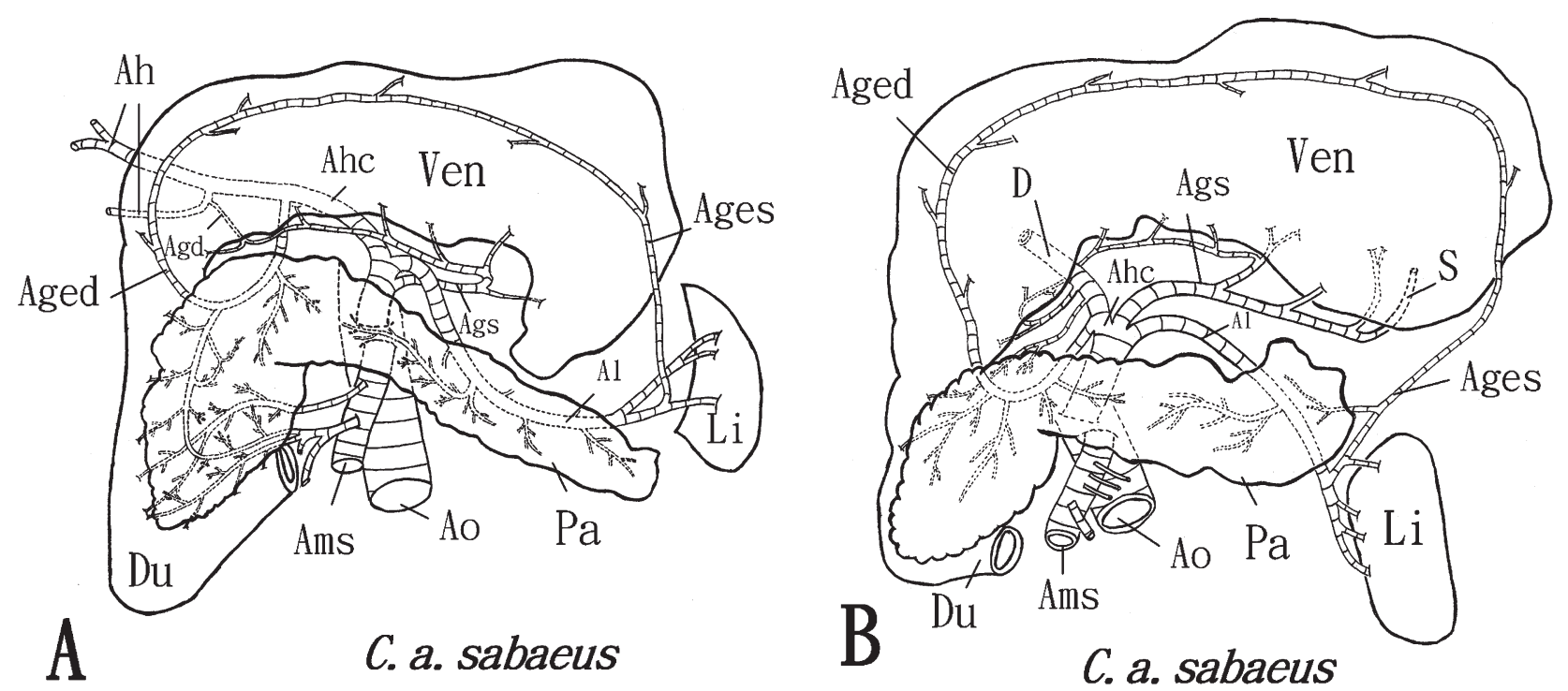

Fig. 7. The arterial distribution to the pancreas of the Cercopithecus aethiops sabaeus (two out of ten cases).

7-A: One case in which the pancreas received both the celiac and the superior mesenteric arteries, although the distribution by the superior mesenteric was minor. 7-B: the case in which the pancreas received only the celiac distribution.
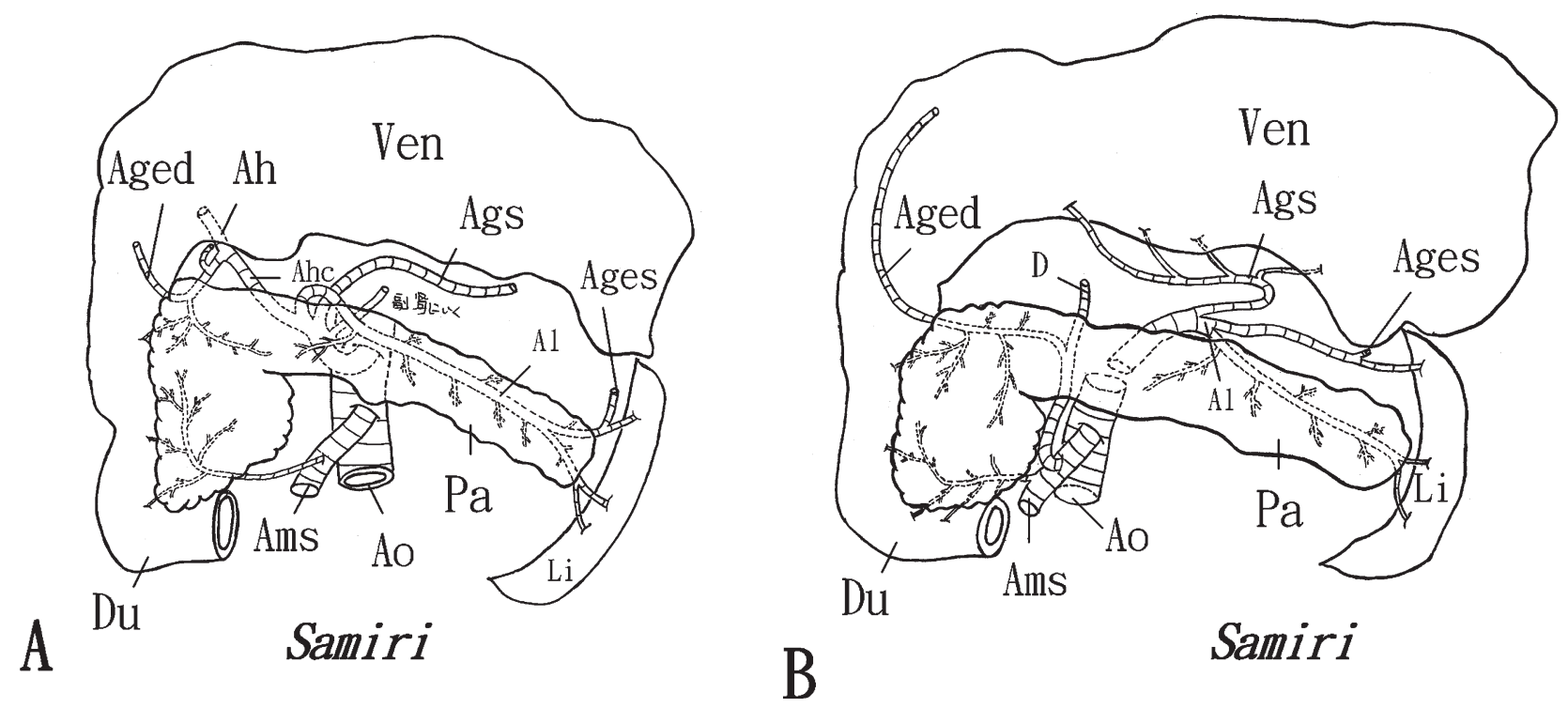

Fig. 8. The arterial distribution to the pancreas of the Saimiri. Two out of the six cases).

8-A: The celiac artery delivered the left gastric, the splenic, the common hepatic, and the right adrenal arteries. The pancreas received both celiac and superior mesenteric arteries, although the region covered by the superior mesenteric artery was small. 8-B: One case out of the six saimiri examined. The celiac artery defects the gastroduodenal artery. The right gastroepiploic artery oriinated from the superior mesenteric artery.

gastroepiploic artery originated from the splenic in the five cases, and the right gastroepiploic originated from the superior mesenteric and the left gastroepiploic originated from the splenic artery in one case (Fig. 8).

The pancreas received blood supply from the celiac artery and the superior mesenteric arteries in all six saimiris. This blood distribution was classified in two patterns. (1) one part of the pancreas head was supplied by the superior mesenteric artery, while the other part of the pancreas was supplied by the celiac artery (five 
cases among them). (2) in one case, all part of pancreas head was supplied by the superior mesenteric artery via a direct communication or via the right gastroepiploic artery. The pancreas body and the tail were supplied by the splenic artery with the direct communication (Fig. 8).

\section{Discussion}

1. Area covered by the celiac artery and the superior mesenteric artery in the pancreas.

In humans, it is thought that there are three types of the arterial distribution to the pancreas (Miyaki T, 2000) (Fig. 9). Type I: The celiac artery supplied the pancreas tail, body and the head. But a part of the pancreas head was supplied by the superior mesenteric artery. Type II: The superior mesenteric artery supplied to a part of the pancreas body and the half of the pancreas head. Type III: The pancreas was equally supplied by the celiac artery and by the superior mesenteric artery (Fig. 9).

In this study with apes, the arterial distribution was
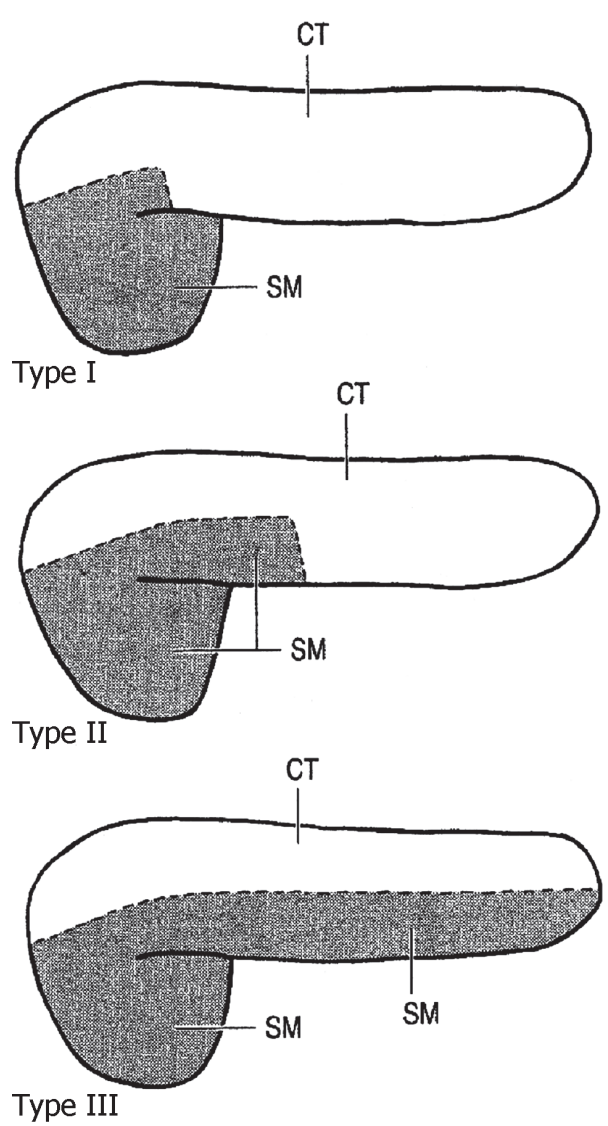

Fig. 9. The area covered by the celiac and the superior mesenteric artery in humans (quote from the reference by Miyaki, 2000). There are three types reported. CT: the area covered by the celiac artery. SM: the area covered by the superior mesenteric artery. found more varied than in humans. The patterns of the arterial distribution to the pancreas in the anthropoids, catarrhine monkey, and the platyrrhine monkey are shown in Fig. 10. In this figure, the left column shows the distribution patterns in anthropoids. In anthropoids, the distribution was very much varied. In a white-handed gibbon, the pancreas was completely supplied by the celiac artery. In the two chimpanzees, the superior mesenteric artery supplied almost all region of the pancreas. These patterns are not seen in humans.

In the middle column in Fig. 10, the patterns of the catarrhine monkeys are shown. In one anubis baboon and in one savannah monkey, the whole pancreas was supplied by the celiac artery. Generally, the supply from the superior mesenteric artery was limited in the cararrhine monkey in our study. In the right column in Fig. 10 , the patterns of the platyrrhine monkey are shown. There were two types of the distribution patterns in the platyrrhine monkey. Although the pancreas was always supplied by both celiac and the superior mesenteric arteries, the whole pancreas head was supplied by the superior mesenteric artery in one case.

As the whole monkey species, we summarized the patterns of the arterial distribution to the pancreas in the monkey (Fig. 11). There were two categories of arterial distribution, and six types. Category A: the celiac artery and the superior mesenteric artery both provided the blood supply to the pancreas. Within this category, there were five types:

Category A-type a: a small part of the pancreas body and the lower half of the head were supplied by the superior mesenteric artery. The rest of the pancreas was supplied by the celiac artery (the case of gorilla).

Category A-type b: the celiac artery supplied almost all parts of the pancreas except for a small region in the pancreas head, where the superior mesenteric artery provided the supply (chimpanzee 1, white-handed gibbon1, hamadryas baboon, anibus baboon 2, savannah money 1 $-8,10$, and squirrel monkey 1, 3-6).

Category A-Type c: the pancreas head was supplied by the superior mesenteric artery. The body and the tail were supplied by the celiac artery (squirrel monkey 2).

Category A-Type d: a small part of the pancreas head and of the tail were supplied by the celiac artery. The pancreas body was supplied by the superior mesenteric artery (chimpanzee 2).

Category A-Type e: a small part of the pancreas head was supplied by the celiac artery. Most of the pancreas was supplied by the superior mesenteric artery. (Chimpanzee 3).

Category B: the whole of the pancreas was supplied by the celiac artery (white-handed gibbon 2 in Fig. 4, anubis baboon 1 in Fig. 6, savannah monkey B in Fig. 7) (Shawuti A et al., 2007, 2008a, 2008b). This category was not seen in humans. We adopted the names: category and type to compare the types between the apes and 
(1)

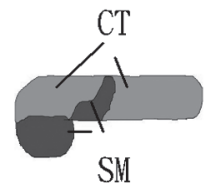

(2)

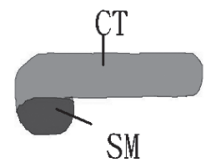

(3)

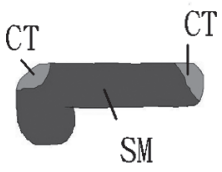

(4)<smiles>[GeH3]</smiles>

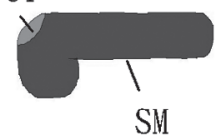

(5)

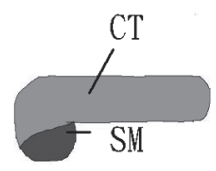

(6)

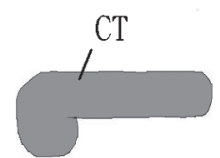

(1)

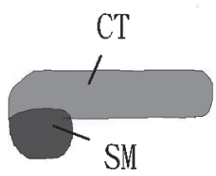

(2)

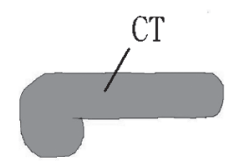

(3)

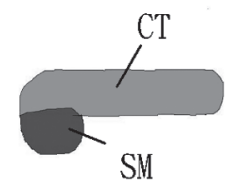

(4)

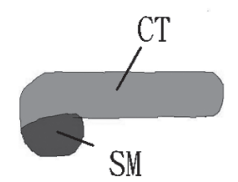

(5)

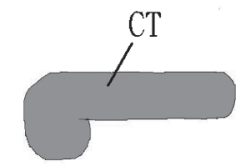

(1)

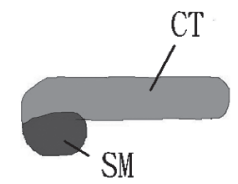

(2)

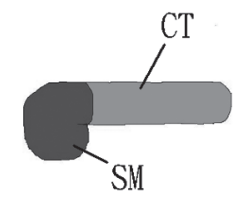

Fig. 10. The area covered by the celiac and/or superior mesenteric artery in the antropoids, catarrhine monkeys, and the platyrrhine monkeys.

(1): Gorilla, (2)-(4): chimpanzees, (5)-(6): white-handed gibbons in the left column.

(1): hamadryas baboon, (2)-(3): anubis baboons, (4)-(5): savannah monkeys in the middle column.

(1)-(2): squirrel monkeys in the right column.

There were nine savannah monkeys which showed the same pattern (4) in the middle column. Also, five squirrel monkeys showed the same pattern. They were summarized in (1) in the right column.

humans in the figures 9 and 11.

Larsen and Tooyama described that the human pancreas develops from the endoderm of the caudal portion of the foregut (Larsen WJ, 1999; Tooyama et al., 2003). At the end of the fourth week of embryo, a protrusion of a part of the endoderm in the dorsal duodenum proceeds to the dorsal pancreas bud. Within three days after this, the endodermal enlargement at the orifice of the bile duct becomes the ventral pancreas bud. In the fifth week of embryo, the ventral pancreas bud moves to the dorsal side with the bile duct by the rotation of the duodenum. Before the sixth week, the two pancreatic buds will be united to become one pancreatic body. After studies, Kleitsch concluded that the dorsal pancreatic bud will receive the blood supply from the celiac artery (Kleitsch WD, 1955; Kirk, 1931; Oierson JM, 1943). The ventral pancreatic bud receives from the superior mesenteric artery. We think that the arterial distribution to the pancreas in the primates would have a close comparative relationship to the development of the human body. The dorsal pancreas bud is supplied by the celiac artery. The ventral pancreas bud is supplied by the superior mesenteric artery. After the unity of both buds, the pancreas became supplied by both celiac and superior mesenteric arteries.

In this study, we found that the pancreas was supplied by both arteries basically; in the gorilla, chimpanzee 1-3, the white-handed gibbon, the hamadryas baboon, anubis baboon 1, savannah monkey (nine out of ten subjects), and in all squirrel monkeys (Fig. 10). We also found that there were two patterns of exception. One pattern was that the superior mesenteric artery supplied almost all parts of the pancreas and that the celiac artery supplied only a small part of the pancreas (chimpanzee $\mathrm{B}$ and $\mathrm{C}$ in 

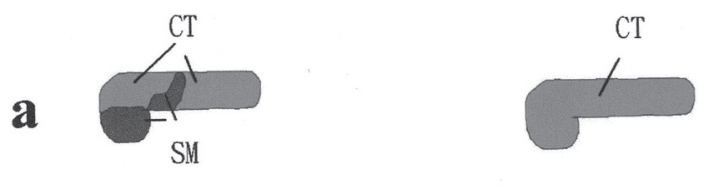

$b+\frac{\mathrm{CT}}{\mathrm{SM}}$
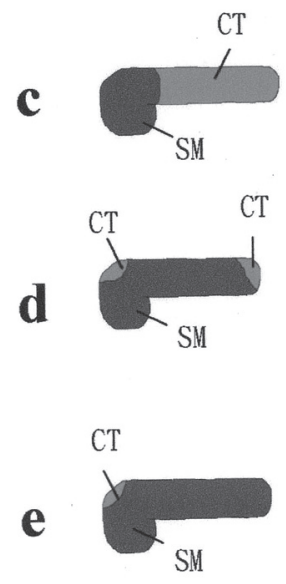

\section{Category A}

Fig. 11. The summarizing figure of the arterial distribution to the pancreas of the apes.

Category A: the patterns of the arterial distribution in which both celiac and superior mesenteric arteries distributed. There were five types in this category.

Category B: the pancreas to which only the celiac artery supplied.

Fig. 3). The other pattern was that the celiac artery supplied the whole pancreas. (white-handed gibbon 2, anubis baboon 1, savannah monkey 9). (Figs. 4, 6, 8, 10).

\section{The right gastroepiploic artery.}

In humans, it is thought that the right gastroepiploic artery originates from the celiac artery. However, in the apes, in the six subjects out of 25 apes showed that the right gastroepiploic artery originated from the superior mesenteric artery. In these cases, the gastroduodenal artery disappeared. Because the right gastroepiploic artery received blood supply through the anastomosis between the inferior pancreaticoduodenal artery and the superior pancreaticoduodenal artery, the right gastroepiploic artery originated from the periphery of the superior mesenteric artery. After the study with apes, we hypothesize that the right gastroepiploic artery may originate from the superior mesenteric artery in humans. The gastroduodenal artery exists unstably in the embryological development.

\section{Conclusion}

The pancreas was supplied by both the celiac and the superior mesenteric artery. Theoretically, the pancreas can be supplied by three types; (1) by both arteries, (2) by the celiac artery alone, and (3) by the superior mesenteric artery alone. In this study with apes, we could find all types, although the supply by the superior mesenteric artery alone (type (3) was not so complete. Therefore, we think that it is highly possible to see types (2) and (3) in humans. The existence of the types (2) and (3) means that the arterial distribution to the pancreas progressed independently to the development of the pancreatic buds.

The right gastroepiploic artery originated from the superior mesenteric artery in chimpanzees, in all papios, and in a squirrel monkey. The right gastroepiploic artery which originated from the superior mesenteric artery is also anticipated in humans.

\section{References}

1) Akaiwa J. About the arterial distribution to the head part of the pancreas in Japanese fetuses. J Nippon Med School 1984; 51:182 -191 (in Japanese with English abstract).

2) Shawuti A, Miyaki T, Kumakura $H$ and Itoh M. The arterial distribution to the pancreas in the Gorilla, chimpanzees and whitehanded gibbons. Keitai Kagaku 2007; 10(2):77-82 (in Japanese).

3a) Shawuti A, Miyaki T, Saito T and Itoh M. The arterial distribution to the pancreas of the three species in the catarrhine monkey. Keitai Kagaku 2008; 12(1):27-31 (in Japanese).

3b) Shawuti A, Miyaki T, Saito T and Itoh M. The arterial supply to the pancreas in the platyrrhine monkey, saimiri. Keitai Kagaku 2008; 12(1):33-35.

4) Gaujoux S, Sauvanet A, Vullierme MP, Cortes A, Dokmak S, Sibert A, Vilgrain V and Belghiti J. Ischemic Complications after Pancreaticoduodenectomy. Annals of Surgery 2009; 249(1):111117.

5) Gray's Anatomy. Fortieth ed. of Gray's Anatomy ed. by Standring S et al. Churchill Livingstone, 20008, 1183-1190.

6) Hashimoto K. The structure and the function of the pancreas (in Japanese). Uchuu-do Yagi publishing Co., 2003; pp. 12-15.

7) Iwamoto M. Lecture: The classification of the Monkey (1. Macaccu). Reichow-rui kenkyuu 1985; 1:45-54 (in Japanese).

8) Iwamoto M. Lecture: The classification of the Monkey (4. Anthropods). Reichow-rui kenkyuu 1987; 3:119-126 (in Japanese).

9) Kato Y. Comparative Anatomy of the Domestic Animals, 6th ed. (in Japanese), ed. by Yoshitaro Kato, Youken-dow publishing Co., Tokyo, Japan, 1964.

10) Kirk E. Untersuchung ueber die groebere und feinere topographische Verteilung der Arterien, Venen und Ausfuehrungsgaenge in der menschlischen Bauchspeichel druese, Ztschr $\mathrm{f} d$ ges Anat. (Abt 1) 1931; 94:875-882.

11) Kleitsch WD. Anatomy of the Pancreas. AMA Archives of Surgery $1955 ; \mathbf{7 1 ( 6 ) : 7 9 5 - 8 0 2 . ~}$

12) Larsen WJ. Saishin Jintai Hasseigaku, 2nd ed. translated by Aikawa E., Yamashita K., Miki A., Ootani H.: Nishimura publishing Co. (in Japanese) 1999; pp. 206-219.

13) Martin II RG, Scoggins CR, Stanley CA, McMasters KM and Kooby DA. Arterial and Venous Resection for Pancreatic Adenocarcinoma. Operative and long-term outcomes. Arch Surg 2009; 144(2):154-159.

14) Miyaki T. Visceral branches of the abdominal aorta: Hepatic artery, Biliary artery and Pancreatic artery. In "Anatomic varia- 
tions in Japanese.” ed by Sato T, Akita K, 2000; pp. 242-249, 352 -360 . University of Tokyo Press.

15) Nomina Anatomica Veterinaria Japonica, ed. by the Japanese Society of Veterinary Anatomists. Kyoei trading Co. 1978; Tokyo Japan.

16) Oierson JM. The arterial blood supply of the pancreas. Surg Gynec \& Obst 1943; 77:426-432.

17) Sakagami S. Distribution of pancreatic arteries in the human fetus. J Nippon Med School 1989; 56(4):365-382 (in Japanese with English abstract).

18) Swindler DR and Wood CD. An atlas of primate gross anatomy, Baboon, Chimpanzee, and Man. 1973; pp. 212-219, University of Washington Press. Seattle.

19) Tooyama T, Ootsuka $M$ and Nakajima Y. Jintai Hassei Gaku (in Japanese) Nanzando, 2003; pp. 188-193.

20) Uchida T. Yazu-Uchida Dictionary of the Animal Classification. Nakayama book Publishing Co. Ltd., Tokyo, Japan, 1988. 